DOI: https://doi.org/10.24297/jam.v20i.8912

\title{
ON POINTWISE PRODUCT VECTOR MEASURE DUALITY
}

Levi Otanga Olwamba ${ }^{1}$ and Maurice Owino Oduor ${ }^{2}$

Department of Mathematics and computer science

University of Kabianga

\begin{abstract}
:
This article is devoted to the study of pointwise product vector measure duality. The properties of Hilbert function space of integrable functions and pointwise sections of measurable sets are considered through the application of integral representation of product vector measures, inner product functions and products of measurable sets.
\end{abstract}

Keywords: Measurable sets, Vector measure duality, Integrable functions.

\section{Introduction}

Over the years, mathematics scholars have studied inner product functions in Banach spaces. In previous results, many theories on integration of vector valued functions with respect to vector measure duality have been proved. This paper explores pointwise projections and sections of measurable sets. The study proves the existence of integral representation of pointwise product vector measure duality with values in a Hilbert space. Throughout this paper, $\left(\mu_{i}\right)_{i \in I}$ and $\left(\nu_{i}\right)_{i \in I}$ denote families of set functions indexed by a finite set $I$. The functions $\left(\mu_{i}\right)_{i \in I}$ and $\left(\nu_{i}\right)_{i \in I}$ defined on sigma rings $\rho$ and $\varepsilon$ with values in Hilbert spaces $X$ and $Y$ respectively i.e $\mu_{i}: \rho \rightarrow X$ and $\nu_{i}: \varepsilon \rightarrow Y$ for each $i \in I$, are called vector measures. The function $\mu_{i} \times \nu_{i}: \rho \times \varepsilon \rightarrow X \times Y$ denotes the product of vector measures $\mu_{i}: \rho \rightarrow X$ and $\nu_{i}: \varepsilon \rightarrow Y$ . The function $\left(T_{t \mu_{i} \times \nu_{i}}\right)_{i \in I}$ denotes a family of non-negative vector measures in $M(\rho \times \varepsilon, X \times Y)$, where $T_{t}$ is an integrable vector valued function with respect to a vector measure $\nu_{i}$ for each $i \in I$. There exists a vector measure function $g_{i}^{t}$ for $t \in \mathbf{R}$ in $M(\rho \times \varepsilon, X \times Y)$, where $M(\rho \times \varepsilon, X \times Y)$ is a set of $X \times Y$ valued vector measures defined on $\rho \times \varepsilon$.

\section{Basic concepts}

Definition 1 (Okada et al.,2008, Otanga et al., 2015a, Yaogan, 2013)

If $g_{i}^{t} \leq T_{t \mu_{i_{k}} \times \nu_{i_{k}}}$ for $i<k_{i}$ is an increasingly directed family of vector

measures, we define the function $g_{i}^{t}$ by

$$
g_{i}^{t}(A \times B)=L U B_{i_{k}} T_{t_{\mu_{i_{k}} \times \nu_{i_{k}}}}(A \times B)
$$

where $i, i_{k} \in I, t \in \mathbf{R}$ and $A \times B$ is a fixed measurable set with respect 
to $\rho \times \varepsilon$.

If $\psi^{(A \times B)}$ is a vector valued function, where $A \times B \in \rho \times \varepsilon$ is a fixed set,

then the product of $\psi^{(A \times B)}$ and $T_{t \mu_{i_{k}} \times \nu_{i_{k}}}$ is given by

$$
\psi^{(A \times B)} * T_{t \mu_{i_{k}} \times \nu_{i_{k}}}=T_{t \mu_{i_{k}} \times \nu_{i_{k}}}(A \times B) \in X \times Y
$$

If $b \in B$ is a fixed element, then the set $(A \times B)^{b}$ is measurable with

respect to $\rho$. Therefore,

$$
L U B_{i_{k}} \psi^{(A \times B)^{b}} * T_{t \mu_{i_{k}}}=g_{i}^{t}\left((A \times B)^{b}\right)=L U B_{i_{k}} T_{t} \mu_{i_{k}}(A \times B)^{b} \in X
$$

For each $i \in I$ and $G \subset X \times Y$, we define

$\nu_{i} * T_{t} \mu_{i}\left((A \times B)^{b}\right)=\int T^{t} \mu_{i}\left((A \times B)^{b}\right) \delta \nu_{i} \in G$.

Definition 2 (Dorlas, 2010 and Otanga, 2015a)

If $Q \in \rho \times \varepsilon$, then the set $Q^{b}=(A \in \rho: A \times B \subset Q)$ where $b \in B$ and $A$

is a fixed set of finite measure, is called a fixed segment of the set $Q$.

Definition 3 (Rodriguez, 2006)

If $T_{t} \mu_{i} \times \nu_{i}: \rho \times \varepsilon \rightarrow X \times Y$ is a product vector measure, then the integral of the function $T_{t}$ with respect to the pointwise product vector measure duality is given by $<\int T_{t} \mu_{i}\left((A \times B)^{b}\right) \delta \nu_{i}, g^{*}>$, where $A \times B$ is a fixed measurable set with respect to $\rho \times \varepsilon, b \in B, g^{*}$ is an element in $G^{*}$, the dual space of the Hilbert space $G$. 
Definition 4 (Otanga et al., 2015b and Sanchez, 2004)

Let $T_{t} \mu_{i} \times \nu_{i} \in M(\rho \times \varepsilon, X \times Y)$. Consider a fixed element $b \in B$ and the integral map $\psi_{g^{*}}^{(A \times B)}$ where $g^{*} \in G^{*}$. The pointwise integral of the function $T_{t}$ generated by $\psi_{g^{*}}^{(A \times B)}$ is given by

$$
\psi_{g^{*}}^{(A \times B)^{b}}\left(\nu_{i} * T_{t} \mu_{i}\right)=<\int T_{t} \mu_{i}\left((A \times B)^{b}\right) \delta \nu_{i}, g^{*}>
$$

\section{Results}

The following propositions are devoted to analyse pointwise integral repre-

sentation of the product vector measure $\nu_{i} * T_{t} \mu_{i}$ and its relationship with

the inner product vector measure duality denoted by

$<\int T_{t} \mu_{i}\left((A \times B)^{b}\right) \delta \nu_{i}, g^{*}>$ for each $i \in I$, where $t \in \mathbf{R}, A \times B$ is a

measurable set with respect to $\rho \times \varepsilon, b \in B$ is a fixed element and $g^{*}$ is

an element in $G^{*}$, the dual space of the Hilbert space $G$.

\section{Proposition 1}

Let $\left(\Phi, \rho \times \varepsilon, \mu_{i} \times \nu_{i}\right)$ for each $i \in I$ be a measure space, where $\Phi$ is a

non-empty two dimensional set and $\psi^{\left(\mu_{i} \times \nu_{i}\right)} \in M(\rho \times \varepsilon, X \times Y)$. Then

$\int \psi\left(T_{t} \mu_{i}\right) \delta \nu_{i}=\psi\left(\nu_{i} * T_{t} \mu_{i}\right)$ for each $i \in I$ and $t \in \mathbf{R}$

\section{Proof}


Let $\psi_{g^{*}}^{(A \times B)^{b}}$ be an integral function, where $A \times B \in \rho \times \varepsilon, g^{*} \in G^{*}$ and

$b \in B$ is a fixed element. As a consequence of integral representation of

the product vector measure $\nu_{i} * T_{t} \mu_{i}$ as illustrated in (Otanga et al., 2015a

and Yaogan, 2013), we obtain

$$
\begin{aligned}
\int \psi_{g^{*}}^{(A \times B)^{b}}\left(T_{t} \mu_{i}\right) \delta \nu_{i}= & <\int\left(T_{t} \mu_{i}\right)(A \times B)^{b} \delta \nu_{i}, g^{*}>=<\left(\nu_{i} * T_{t} \mu_{i}(A \times B)^{b}, g^{*}>\right. \\
& =\psi_{g^{*}}^{(A \times B)^{b}}\left(\nu_{i} * T_{t} \mu_{i}\right)
\end{aligned}
$$

Therefore, $\int \psi\left(T_{t} \mu_{i}\right) \delta \nu_{i}=\psi\left(\nu_{i} * T_{t} \mu_{i}\right)$ for each $i \in I$ and $t \in \mathbf{R}$

\section{Proposition 2}

Let $\left(\mu_{i}\right)_{p_{i}}^{p_{j}}=T_{s}\left(\mu_{i}\right) p_{p_{i}}^{p_{j}} s \in \mathbf{R}$. Then $\nu_{i} *\left(T_{t}\left(\mu_{i}\right)_{p_{i}}^{p_{j}}\right)=T_{t}\left(\nu_{i} * \mu_{i}^{p_{i}}\right)$, where

$p_{i}$ and $p_{j}$ are measurable sets of finite measure, $p_{i} \subset p_{j}$ for each $i, j \in I$,

$i \neq j$ and $s, t \in \mathbf{R}$.

\section{Proof}

Let $A \times B$ be a measurable set with respect to $\rho \times \varepsilon$ and $g^{*} \in G^{*}$. For a

fixed element $b \in B$, the set $(A \times B)^{b}$ is measurable with respect to $\rho$. It

follows that

$<\nu_{i} *\left(T_{t}\left(\mu_{i}\right)_{p_{i}}^{p_{j}}\right)^{(A \times B)^{b}}, g^{*}>=<\int T_{t+s} \mu_{i}^{p_{j}}\left(p_{i} \cap(A \times B)^{b}\right) \delta \nu_{i}, g^{*}>$ for $s, t \in \mathbf{R}$

Applying the results in (Otanga, 2015b and Otanga et al., 2015b) on

contraction of a vector measure $\mu_{i}$ by $p_{i}$ for each $i \in I$, we obtain 
$<\int T_{t+s} \mu_{i}\left(p_{i} \cap p_{j} \cap(A \times B)^{b}\right) \delta \nu_{i}, g^{*}>=<\int T_{t+s} \mu_{i}\left(p_{i} \cap(A \times B)^{b}\right) \delta \nu_{i}, g^{*}>$

$\left.=<\int T_{t+s}\left(\mu_{i}\right)^{p_{i}}(A \times B)^{b}\right) \delta \nu_{i}, g^{*}>=\int \psi_{g^{*}}^{(A \times B)^{b}}\left(T_{t}\left(T_{s} \mu_{i}^{p_{i}}\right) \delta \nu_{i}\right.$

$=\int T_{t} \psi_{g^{*}}^{(A \times B)^{b}}\left(T_{s} \mu_{i}^{p_{i}}\right) \delta \nu_{i}$. Since $\int T_{s} \mu_{i}^{p_{i}} \delta \nu_{i}=\nu_{i} * T_{s} \mu_{i}^{p_{i}}$, it follows that

$\left.\int T_{t} \psi_{g^{*}}^{(A \times B)^{b}}\left(T_{s} \mu_{i}^{p_{i}}\right) \delta \nu_{i}=T_{t}\left(\psi_{g^{*}}^{(A \times B)^{b}}\right)\left(\nu_{i} * T_{s} \mu_{i}^{p_{i}}\right)\right)$. On application of the

relation $T_{s} \mu_{i}^{p_{i}}=\mu_{i}^{p_{i}}$ for $i \in I$ and $s \in \mathbf{R}$, we obtain

$\left.T_{t}\left(\psi_{g^{*}}^{(A \times B)^{b}}\right)\left(\nu_{i} * T_{s} \mu_{i}^{p_{i}}\right)\right)=\psi_{g^{*}}^{(A \times B)^{b}}\left(T_{t}\left(\nu_{i} * \mu_{i}^{p_{i}}\right)\right)$

$$
=<T_{t}\left(\nu_{i} * \mu_{i}^{p_{i}}\right)(A \times B)^{b}, g^{*}>\text {. }
$$

In general, $\nu_{i} *\left(T_{t}\left(\mu_{i}\right)_{p_{i}}^{p_{j}}\right)=T_{t}\left(\nu_{i} * \mu_{i}^{p_{i}}\right)$.

\section{Proposition 3}

Let $\psi_{g^{*}}$ represent an integral function with respect to vector measure

duality and $\mu_{i} \times \nu_{i} \times \alpha_{i}$ for each $i \in I$, be a vector measure defined on

$\rho \times \varepsilon \times \tau$. If $<\int\left(T_{t} \mu_{i}\right) \delta \nu_{i}, g^{*}>=\psi_{g^{*}}\left(\nu_{i} * T_{t} \mu_{i}\right)$ for each $g^{*} \in G^{*}$, then

$\left(\alpha_{i} * \nu_{i}\right) * T_{t} \mu_{i}=\alpha_{i} *\left(\nu_{i} * T_{t} \mu_{i}\right)$ for $t \in \mathbf{R}$

\section{Proof}

Let $A \times B \times C \in \rho \times \varepsilon \times \tau$ and $g^{*} \in G^{*}$. Application of the Integral function

$\left(\psi_{g^{*}}\right)^{(A \times B \times C)}$ as illustrated in (Campo et al., 2010), gives

$\left(\psi_{g^{*}}\right)^{(A \times B \times C)}\left(\alpha_{i} \times \nu_{i}\right) * T_{t} \mu_{i}=<\iint\left(T_{t} \mu_{i}\right)(A \times B \times C) \delta \alpha_{i} \delta \nu_{i}, g^{*}>$

Suppose $(b, c) \in B \times C$ is a fixed point. The set $(A \times B \times C)^{(b, c)}$ is 
measurable with respect to $T_{t} \mu_{i}$. It follows that

$$
\begin{gathered}
\left(\psi_{g^{*}}\right)^{(A \times B \times C)^{(b, c)}}\left(\alpha_{i} \times \nu_{i}\right) * T_{t} \mu_{i}=<\iint\left(T_{t} \mu_{i}\right)(A \times B \times C)^{(b, c)} \delta \alpha_{i} \delta \nu_{i}, g^{*}> \\
=<\iint\left(T_{t} \mu_{i}\right)(A)^{(b, c)} \delta \alpha_{i} \delta \nu_{i}, g^{*}>
\end{gathered}
$$

If $b \in B$ is a fixed element, then the set $A \times B \times C$ is projected onto $A \times C$.

Therefore,

$$
\begin{aligned}
\left(\psi_{g^{*}}\right)^{(A \times B \times C)^{(b, c)}}\left(\alpha_{i} \times \nu_{i}\right) * T_{t} \mu_{i}=< & \int\left(\alpha_{i} * T_{t} \mu_{i}\right)(A \times C)^{b} \delta \nu_{i}, g^{*}> \\
& =\left(\psi_{g^{*}}\right)^{(A \times B \times C)^{(b, c)}}\left(\nu_{i} *\left(\alpha_{i} * T_{t} \mu_{i}\right)\right)
\end{aligned}
$$

If $c \in C$ is a fixed element, then the set $A \times B \times C$ is projected onto

$A \times B$ (Dorlas, 2010). Therefore,

$$
\begin{aligned}
\left(\psi_{g^{*}}\right)^{(A \times B \times C)^{(b, c)}}\left(\alpha_{i} \times \nu_{i}\right) * T_{t} \mu_{i}=< & \int\left(\nu_{i} * T_{t} \mu_{i}\right)(A \times B)^{c} \delta \alpha_{i}, g^{*}> \\
& =\left(\psi_{g^{*}}\right)^{(A \times B \times C)^{(b, c)}}\left(\alpha_{i} *\left(\nu_{i} * T_{t} \mu_{i}\right)\right)
\end{aligned}
$$

Hence, the above relation gives $\left(\alpha_{i} \times \nu_{i}\right) * T_{t} \mu_{i}=\alpha_{i} *\left(\nu_{i} * T_{t} \mu_{i}\right)$

\section{Proposition 4}

For a fixed $b \in B$ and for all $t \in \mathbf{R}$, let $g_{i}^{t}(A \times B)^{b} \in X$ such that

$g_{i}^{t} \leq T_{t} \mu_{i_{k}}$ for $i<i_{k}$ and each $i, i_{k} \in I$. If $P^{(X \times Y)}: \mathbf{R} \rightarrow M(\rho \times \varepsilon, X \times Y)$

is an integrable function with respect to $\mu_{i} \times \nu_{i}$, then for a fixed element

$x \in X, P_{t}^{(X \times Y)^{x}} * g_{i}^{t}=L U B_{i_{k}} P_{t}^{y} * T_{t} \mu_{i_{k}}$ for $\forall y \in Y$

\section{Proof}


Let $A \times B \in \rho \times \varepsilon$, fix $b \in B$ and $x \in X$. For every element $y \in Y$, we have

$\psi_{g^{*}}^{(A \times B)^{b}}\left(\nu_{i} * P_{t}^{(X \times Y)^{x}} * g_{i}^{t}\right)=\psi_{g^{*}}^{(A \times B)^{b}}\left(\nu_{i} * P_{t}^{y} * g_{i}^{t}\right) \forall y \in Y$

The product of vector measures $\left(\nu_{i} * P_{t}^{y}\right)$ and $g_{i}^{t}$ in $M(\rho \times \varepsilon, X \times Y)$ and the application of the integral function $\psi_{g^{*}}^{(A \times B)^{b}}$ (Rodriguez, 2006 and Yaogan, 2013), implies that

$\psi_{g^{*}}^{(A \times B)^{b}}\left(\nu_{i} * P_{t}^{y} * g_{i}^{t}\right)=<\int g_{i}^{t}(A \times B)^{b} P_{y}^{t} \delta \nu_{i}, g^{*}>$

Since $g_{i}^{t} \leq T_{t} \mu_{i_{k}}$ for $i<i_{k}$ and $t \in \mathbf{R}$, then by the property of increasingly

directed vector measure duality, we obtain

$$
\begin{aligned}
<g_{i}^{t}(A \times B)^{b} P_{t}^{y} \delta \nu_{i}, g^{*}>= & L U B_{i_{k}}<\int T_{t} \mu_{i_{k}}(A \times B)^{b} P_{t}^{y} \delta \nu_{i}, g^{*}> \\
& =L U B_{i_{k}} \psi_{g^{*}}^{(A \times B)^{b}}\left(\nu_{i} *\left(P_{t}^{y} * T_{t} \mu_{i_{k}}\right)\right)
\end{aligned}
$$

Comparing equations $(*),(* *)$ and $(* * *)$, we obtain

$P_{t}^{(X \times Y)^{x}} * g_{i}^{t}=L U B_{i_{k}} P_{t}^{y} * T_{t} \mu_{i_{k}}$

\section{Proposition 5}

Let $t_{o}$ and $t$ be real numbers. Let $B$ be a measurable set with respect to

$\varepsilon$ such that $B \downarrow \emptyset$. Let $P^{\nu_{i}(B)} * g_{i}^{t_{o}}=g_{i}^{t_{o}}$ as $\nu_{i}(B) \downarrow 0$ and $g_{i}^{t_{o}} \leq T_{t_{o}} \mu_{i_{k}} \times \nu_{i_{k}}$

for $i<i_{k}$. If $T_{t-t_{O}} T_{t_{o}} \mu_{i_{k}} \times \nu_{i_{k}}=T_{t} \mu_{i_{k}} \times \nu_{i_{k}}$, then

$$
T_{t-t_{O}}\left(g_{i}^{t_{o}}\right)=L U B_{i_{k}} P^{\nu_{i}(B)} * T_{t} \mu_{i_{k}} \times \nu_{i_{k}} \text { as } \nu_{i}(B) \downarrow 0
$$

\section{Proof}


Since $P^{\nu_{i}(B)} * g_{i}^{t_{o}}=g_{i}^{t_{o}}$ as $\nu_{i}(B) \downarrow 0$ by hypothesis, it follows that

$T_{t-t_{O}}\left(g_{i}^{t_{o}}\right)=T_{t-t_{O}}\left(P^{\nu_{i}(B)} * g_{i}^{t_{o}}\right)$ as $\left.\nu_{i}(B) \downarrow 0\right)$. Since $g_{i}^{t_{o}} \leq T_{t_{o}} \mu_{i_{k}} \times \nu_{i_{k}}$

for $i<i_{k}$, by the property of increasingly directed vector measure duality,

it follows that $T_{t-t_{O}}\left(g_{i}^{t_{o}}\right)=T_{t-t_{O}}\left(P^{\nu_{i}(B)} * g_{i}^{t_{o}}\right)$ as $\left.\nu_{i}(B) \downarrow 0\right)$

$=L U B_{i_{k}} T_{t-t_{O}}\left(P^{\nu_{i}(B)} * T_{t_{o}} \mu_{i_{k}} \times \nu_{i_{k}}\right)$ as $\nu_{i}(B) \downarrow 0$

On application of the relation $T_{t-t_{O}} T_{t_{o}} \mu_{i_{k}} \times \nu_{i_{k}}=T_{t} \mu_{i_{k}} \times \nu_{i_{k}}$ (By hypoth-

esis), equation $(*)$ becomes

$L U B_{i_{k}} P^{\nu_{i}(B)} * T_{t-t_{O}} T_{t_{o}} \mu_{i_{k}} \times \nu_{i_{k}}=L U B_{i_{k}} P^{\nu_{i}(B)} * T_{t} \mu_{i_{k}} \times \nu_{i_{k}}$ as $\nu_{i}(B) \downarrow 0$

Hence, $T_{t-t_{O}}\left(g_{i}^{t_{o}}\right)=L U B_{i_{k}} P^{\nu_{i}(B)} * T_{t} \mu_{i_{k}} \times \nu_{i_{k}}$ as $\nu_{i}(B) \downarrow 0$

\section{Proposition 6}

Let $t_{o}$ and $t$ be real numbers, $A \times B \in \rho \times \varepsilon$ and $\mu_{i} \times \nu_{i} \in M(\rho \times \varepsilon, X \times Y)$. If $P^{\nu_{i}(B)} * T_{t} \mu_{i} \times \nu_{i}(A \times B)=T_{t} \mu_{i} \times \nu_{i}(A \times B)$ and $P^{\nu_{i}(B)} * g_{i}^{t_{o}}=g_{i}^{t_{o}}$ as $\nu_{i}(B) \downarrow 0$, then $P^{\nu_{i}(B)} * \mu_{i} \times \nu_{i}=\mu_{i} \times \nu_{i}$ as $\nu_{i}(B) \downarrow 0$

\section{Proof}

By hypothesis, $P^{\nu_{i}(B)} * T_{t} \mu_{i} \times \nu_{i}(A \times B)=T_{t} \mu_{i} \times \nu_{i}(A \times B)$ as $\nu_{i}(B) \downarrow 0$ for $A \times B \in \rho \times \varepsilon$.

Also $T_{t-t_{O}}\left(g_{i}^{t_{o}}\right)=L U B_{i} P^{\nu_{i}(B)} * T_{t} \mu_{i} \times \nu_{i}$ as $\nu_{i}(B) \downarrow 0$ ( By Theorem 5$)$. 
Comparing the two relations, we obtain

$L U B_{i} T_{t} \mu_{i} \times \nu_{i}(A \times B)=T_{t-t_{O}}\left(g_{i}^{t_{o}}(A \times B)\right)$

Suppose $t=\left(\mu_{i} \times \nu_{i}\right)(C \times D)$, where $C \times D \in \rho \times \varepsilon$. Let $C=\emptyset$ or $D=\emptyset$ or

both $C$ and $D$ be empty sets. Considering all the three cases, it follows

that $t=0$. Therefore,

$L U B_{i} \mu_{i} \times \nu_{i}(A \times B)=T_{-t_{O}}\left(g_{i}^{t_{o}}(A \times B)\right)$

Applying $P^{\nu_{i}(B)}$ to the above relation, we obtain

$L U B_{i} P^{\nu_{i}(B)} * \mu_{i} \times \nu_{i}=T_{-t_{O}}\left(P^{\nu_{i}(B)} * g_{i}^{t_{o}}\right)$

By hypothesis, $P^{\nu_{i}(B)} * g_{i}^{t_{o}}=g_{i}^{t_{o}}$ as $\nu_{i}(B) \downarrow 0$. Therefore,

$L U B_{i} P^{\nu_{i}(B)} * \mu_{i} \times \nu_{i}=T_{-t_{O}}\left(g_{i}^{t_{o}}\right)$.

Comparing equations $(*)$ and $(* *)$, we obtain

$P^{\nu_{i}(B)} * \mu_{i} \times \nu_{i}=\mu_{i} \times \nu_{i}$ as $\nu_{i}(B) \downarrow 0$

\section{Corollary}

Let $\left(p_{i}\right)$ be a finite measure defined on $\rho \times \varepsilon$ such that

$\left(p_{i}\right)_{(A \times E)}=\left(\mu_{i} \times \nu_{i}\right)_{(A \times E)}-L U B_{i_{k}} T_{t} \mu_{i_{k}} \geq 0$

for each $i \in I, t \in \mathbf{R}$, where $\left(p_{i}\right)_{(A \times E)}$ and $\left(\mu_{i} \times \nu_{i}\right)_{(A \times E)}$ is the

contraction of $p_{i}$ and $\mu_{i} \times \nu_{i}$ by a measurable set $A \times E \in \rho \times \varepsilon$ of finite

measure. Given $\epsilon>0$, if $\epsilon\left(\mu_{i} \times \nu_{i}\right)_{A \times E} \leq\left(p_{i}\right)_{A \times E}$ and 
$Q^{\nu_{i}(E)} * g_{i}^{t}=L U B_{i_{k}} T_{t} \mu_{i_{k}}$ as $\nu_{i}(E) \downarrow 0$ for a fixed $a \in A$, then $g_{i}^{t} \leq\left(\mu_{i}\right)_{A}$

\section{Proof}

By hypothesis, $\left(p_{i}\right)_{(A \times E)}=\left(\mu_{i} \times \nu_{i}\right)_{(A \times E)}-L U B_{i_{k}} T_{t} \mu_{i_{k}}$. For a fixed

element $e \in E$, let $\left(p_{i}\right)_{A}=\left(\mu_{i}\right)_{A}-L U B_{i_{k}} T_{t} \mu_{i_{k}}$. Since

$Q^{\nu_{i}(E)} * g_{i}^{t}=g_{i}^{t}$ as $\nu_{i}(E) \downarrow 0$ (by proposition 5) and

$Q^{\nu_{i}(E)} * g_{i}^{t}=L U B_{i_{k}} T_{t} \mu_{i_{k}}$ as $\nu_{i}(E) \downarrow 0$ (by hypothesis), it follows that

$g_{i}^{t}=L U B_{i_{k}} T_{t} \mu_{i_{k}}$. For each $\epsilon>0$, let $g_{i}^{t}=\nu_{i} * L U B_{i_{k}} T_{t} \mu_{i_{k}}+\epsilon\left(\mu_{i}\right)_{A}$, where

$A$ is a measurable set of finite measure. Consider the function $\psi_{g^{*}}^{M}$ defined

by $\psi_{g^{*}}^{M}\left(g_{i}^{t}\right)=<g_{i}^{t}(M), g^{*}>$ where $M \in \rho$ and $g^{*} \in G^{*}$, the dual space of

G. Therefore, $\psi_{g^{*}}^{M}\left(g_{i}^{t}\right)=<g_{i}^{t}(M), g^{*}>$

$=L U B_{i_{k}}<\int T_{t} \mu_{i_{k}}(M) \delta_{\nu_{i}}, g^{*}>+<\epsilon\left(\mu_{i}\right)_{A}(M), g^{*}>$

$\leq L U B_{i_{k}}<\int T_{t} \mu_{i_{k}}(M) \delta_{\nu_{i}}, g^{*}>+<\left(p_{i}\right)_{A}(M), g^{*}>$

$=L U B_{i_{k}}\left[<\int T_{t} \mu_{i_{k}}(M) \delta_{\nu_{i}}, g^{*}>+\right.$

$$
\left.\left(<\left(\mu_{i}\right)_{A}(M), g^{*}>-<\int T_{t} \mu_{i_{k}}(M) \delta_{\nu_{i}}, g^{*}>\right)\right]
$$

$=<\left(\mu_{i}\right)_{A}(M), g^{*}>$

In general $g_{i}^{t} \leq\left(\mu_{i}\right)_{A}$ for each $i \in I, t \in \mathbf{R}$ and $A \in \rho$ 


\section{Conclusion}

The results obtained in this paper is a clear demonstration of the existence of integral representation of pointwise product vector measure duality co-domained in a Hilbert space. To this end, the relationship between pointwise integral representation of the product vector measure with the inner product vector measure duality has been revealed.

Acknowledgement: This research was supported by University of Kabianga

\section{REFERENCES}

[1] Campo Del R., Fernandez A., Ferrrando I., Mayoral F. and Naranjo F. (2010). Compactness of multiplication operators on spaces of integrable functions with respect to a vector measure, in vector measure, integration and related topics. Birkhauser Verlag, Basel 201, 109-113.

[2] Dorlas T.C. (2010). Reminder notes for the course on measures on topological spaces. Dublin Institute for Advanced Studies, Dublin, Ireland,10, 1-22.

[3] Okada S., Ricker W. J. and Sanchez P. E. A. (2008). Optimal Domain and Integral Extension of Operators. Birkhauser, Basel, Switzerland,180, 141-148.

[4] Otanga O. L. (2015a). On the Extension of Properties of Cubic Measures. Inter. Math. Forum, Hikari Ltd, 10(3), 105-109.

[5] Otanga O. L. (2015b). On the Finiteness and Sigma Finiteness a Cubic Measure Function. Inter. Math. Forum, Hikari Ltd, 10(3),111-114.

[6] Otanga O. L., Oduor M. O. and Aywa S. O. (2015a). Partition of Measurable Sets. Journal of Advances in Mathematics, 10 (8), 3759-3763.

[7] Otanga O. L., Oduor M. O. and Aywa S. O. (2015b). On Generation of Measurable Covers for Measurable Sets Using Multiple Integral of Functions. Journal of Mathematics and Stastical Science, 2015, 32-41.

[8] Rodriguez J. (2006). On Integration of Vector Functions With Respect to Vector Measures. Czechoslovak Math. J. $\mathbf{5 6}(3), 805-825$.

[9] Sanchez P. E. A. (2004). Vector Measure Duality and Tensor Product Representation of $L_{p}$ - spaces of Vector Measures. Amer. Math. Soc 132, 3319-3326

[10] Yaogan M. (2013). Facts About the Fourier-stieltjes Transform of Vector Measures on Compact Groups. Inter. J. Analy. Appl., 19-25. 